\title{
A General Framework for Spectrum Sensing Using Dedicated Spectrum Sensor Networks
}

\author{
YUNHUAI LIU, Peking University, Beijing Institute of Big Data Research, China \\ QIAN ZHANG, Hong Kong University of Science and Technology, China \\ LIONEL NI, University of Macau, China
}

\begin{abstract}
Efficient spectrum sensing is essential for the successful application of the Dynamic Spectrum Assignment (DSA) technology in Cognitive Radio Networks (CRNs). In conventional spectrum sensing schemes, secondary users (SUs) have to intelligently schedule their sensing and accessing so that the spectrum opportunities are thoroughly exploited while the primary users are not harmed. In this article, we propose a new sensing service model in which a Spectrum Sensor Network (SSN) is employed for spectrum sensing tasks. We will describe the general framework for this SSN-enabled CRN and present the major challenges in such an architecture. We will address one of these challenges and formulate it as a boundary detection problem with unknown erroneous inputs. A novel cooperative boundary detection algorithm is designed which explores recent advances in Support Vector Machines (SVM) and computational geometry. We prove that cooperative spectrum sensing can asymptotically approach the optimal solution. Real testbed as well as comprehensive simulation experiments are conducted, and the results show that, compared with the traditional schemes, cooperative boundary detection can dramatically reduce the spectrum sensing overhead and improve the effectiveness of DSA.
\end{abstract}

CCS Concepts: • Networks $\rightarrow$ Layering;

Additional Key Words and Phrases: Wireless sensor networks, spectrum sensing, dynamic spectrum access

ACM Reference format:

Yunhuai Liu, Qian Zhang, and Lionel Ni. 2019. A General Framework for Spectrum Sensing Using Dedicated Spectrum Sensor Networks. ACM Trans. Sen. Netw. 15, 1, Article 7 (January 2019), 23 pages.

https://doi.org/10.1145/3275244

\section{INTRODUCTION}

Dynamic Spectrum Assignment (DSA) technology brings new hope for alleviating the scarcity of spectrum resources for wireless communications [16, 35]. It allows Secondary Users (SUs) to use a

This research was supported in part by the National Natural Science Foundation of China (NSFC) 61772026 and 61332018 , and National Key R\&D Program of China 2018YFB0803400, the University of Macau Grant SRG2015-00050-FST and by National Basic Research Program of China Grant No. 2014CB340303, Guangdong Natural Science Foundation No. 2017A030312008, and Huawei-HKUST joint lab project.

Authors' addresses: Y. Liu, Peking University, Beijing Institute of Big Data Research, Beijing, China; email: yunhuai.liu@ pku.edu.cn; Q. Zhang, Hong Kong University of Science and Technology, Hong Kong, China; email: qianzh@cse.ust.hk; L. Ni, University of Macau, Macau, China; email: ni@umac.mo.

Permission to make digital or hard copies of all or part of this work for personal or classroom use is granted without fee provided that copies are not made or distributed for profit or commercial advantage and that copies bear this notice and the full citation on the first page. Copyrights for components of this work owned by others than ACM must be honored. Abstracting with credit is permitted. To copy otherwise, or republish, to post on servers or to redistribute to lists, requires prior specific permission and/or a fee. Request permissions from permissions@acm.org.

(C) 2019 Association for Computing Machinery.

1550-4859/2019/01-ART7 \$15.00

https://doi.org/10.1145/3275244 


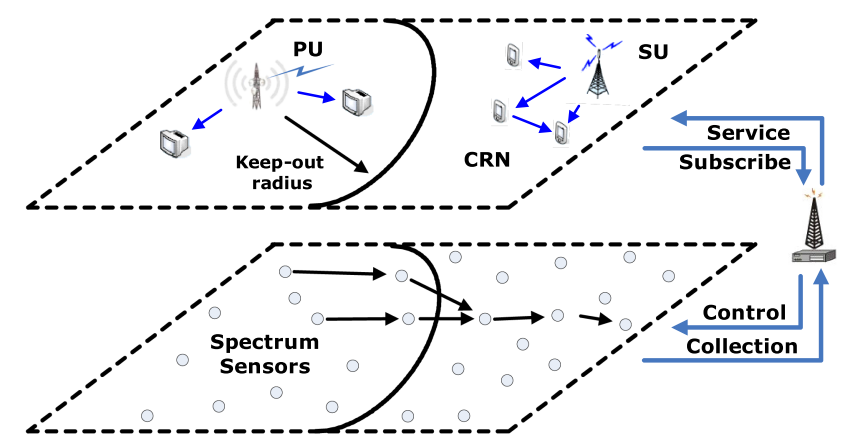

Fig. 1. A general framework of SSN-enabled CRNs. Dedicated spectrum sensors are deployed to provide the spectrum sensing service for SUs, relinquishing the spectrum sensing burden from SUs.

licensed spectrum as long as they cause no harmful interference to spectrum license holders, the Primary Users (PUs) [8]. The spectrum and time period during which SUs can access the spectrum are referred to as spectrum opportunity. By exploiting such opportunities, SUs form Cognitive Radio Networks (CRNs).

In CRNs, a key enabling technology is spectrum sensing. To guarantee the safe usage of PUs, spectrums should be frequently sensed so that PUs' appearance can be instantly detected. When PUs return, SUs should immediately leave the occupied spectrum. In the draft of the IEEE 802.22 standard [29], PUs should be detected within 2 seconds. The probability of misdetection, $P_{M D}$, and false alarms, $P_{F A}$, should be no more than 0.1 . During spectrum sensing, these detectability requirements are mandatory.

In the existing $\mathrm{CRN}$ architecture, both spectrum sensing and accessing are carried out by the SUs themselves. At each time instance, an SU has to make a hard decision: to sense the spectrum availability and check whether the PUs return, or to access the spectrum and explore the spectrum opportunity. This is a dilemma for CRN designers. On one hand, spectrum sensing provides no value for CRN throughput, and thus more time should be allocated to access. On the other hand, necessary sensing is required to guarantee the safety of PU spectrum usage. In addition, because of hardware constraints, individual sensing results are not reliable. Different SUs have to work cooperatively to increase sensing accuracy $[2,41]$.

Indeed, the IEEE 802.22 standard allows the SUs to refer to a database for spectrum availability. This feature motivates us to design a new architecture for CRNs. As illustrated in Figure 1, the spectrum sensing and accessing tasks are separated and carried out by different entities. A dedicated Spectrum Sensor Network (SSN) is employed to undertake the sensing task, store spectrum availability to a central database for reference, and thus release SUs from the sensing burden. We call this architecture $S S N$-enabled CRNs.

In SSN-enabled CRNs, some fundamental issues become obvious. For example, rather than PU's presence at individual locations, we need to know which areas are covered by PUs and which are not. For example, given a limited number of spectrum sensors (e.g., because of the limited budget), what is the optimal sensor deployment and scheduling schemes, when is sensing performance constrained (delay, accuracy, etc.), and how many sensors are needed to achieve the performance?

To answer these questions well, in this article, we give a detailed description of SSN-enabled CRNs and propose our approaches for some of the problems, leaving more for future works. Some skeleton results were reported in our previous conference publication [39], and this article will give a comprehensive analysis. 


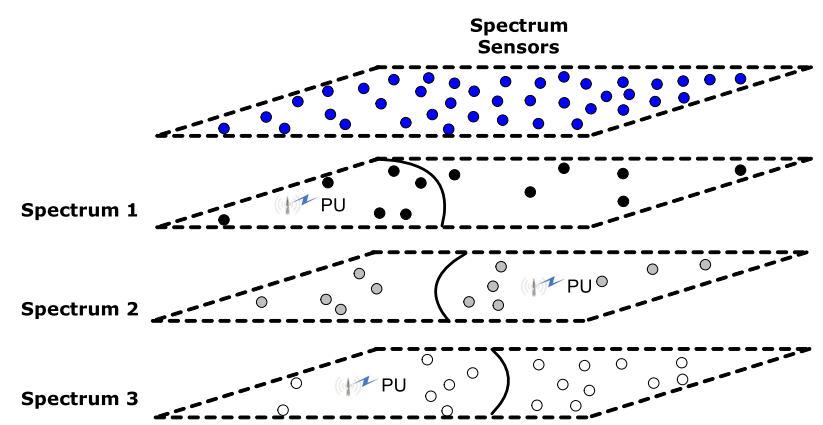

Fig. 2. An illustration of the system model. An SSN is deployed to the field and sensors are assigned and scheduled to different spectrums to accomplish the sensing tasks simultaneously.

The main contributions of this article are as follows.

- We propose a new SSN-enabled CRN architecture. We present the unique features of SSNenable CRNs compared with traditional CRNs and wireless sensor networks. New requirements, issues, and challenges are identified.

- We concentrate on one of the problems and formulate it as a Sensing Error Minimization problem. We prove that the problem has no optimal solution in practical environments and propose a cooperative boundary detection scheme to address the issue.

- We prove that cooperative boundary detection can asymptotically approach the optimal solution in the ideal case. We implement it using off-the-shelf wireless sensor nodes in a prototype system. Comprehensive simulations and field studies show that, compared with the traditional boundary detection algorithms, cooperative boundary detection reduces detection errors by up to $95 \%$, with an average reduction of $85 \%$.

The rest of the article is organized as follows. In Section 2, we describe the architecture of SSNenabled CRNs. In Section 3, we focus on one of the problems and give a formal statement on it. In Section 4, we describe our cooperative boundary approach in detail. In Section 5, we evaluate the performance through simulations and field studies. Related works in the literature are summarized in Section 6. In the last section, we draw a simple conclusion and make some further comments on future work.

\section{SSN-ENABLED CRN ARCHITECTURE}

Figure 2 illustrates the SSN-enabled CRN architecture. In SSN-CRN, a set of spectrum sensors are deployed to the field. They can be large in number with the low cost, well-designed and carefully controlled. At each time instance, sensors are configured to sense a spectrum and declare whether PUs are present. This service can be provided in a centralized manner, in which a central authority delivers the services (as in the Figure 1), or in a fully distributed manner where spectrum sensors themselves deliver the service. SUs simply subscribe to the service and refer to information on their demands. As such, SUs can concentrate on more efficient spectrum access and communications in CRNs [33, 34, 43]. Volunteer SUs (e.g., those with two radios [10]) can also participate in sensing if they agree to function as a simple spectrum sensor.

For spectrum sensors, various sensing techniques can be applied. The state-of-art sensorless sensing technologies can even obtain the mechanical vibration periods with COTS RFID systems [38]. While different sensing techniques may exhibit different sensing characteristics, in SSN, we are more interested in the system's behavior rather than that of individual sensors. 
Basically, during the SSN design, we are subject to the following constraints.

- Budget: For practical concerns, we usually have a limited budget and thus the number of sensors is constrained.

- Frequency: Current hardware advances support frequency hopping within a certain range. Spectrum sensors can easily switch from one spectrum to another nearly instantly. A sensor can sense one spectrum at a time only.

- Sensing accuracy: The sensing should follow the standards so that PUs will not be harmfully affected. For example, IEEE $802.22 \mathrm{draft}$ [24] suggests that sensing delay be no more than 2 seconds with $P_{F A} \leq 0.1$ and $P_{M D} \leq 0.1$. Note that in conventional spectrum sensing, we only care about individual locations where the SUs reside. In SSN, all areas covered by the SSN should be included, as SUs subscribing the service can be anywhere.

- Communication: Spectrum sensors need communications to cooperatively accomplish the sensing task. The communications can be wired or wireless; this issue is beyond the scope of our discussion.

Under these constraints, in SSN-enabled CRNs, there are some new issues that may need further research efforts.

- A first problem is the spectrum sensing algorithm design using spectrum sensors. Given a set of sensors assigned to a spectrum, we are seeking algorithms that can obtain the most accurate sensing results. Recall that, in addition to individual locations, we care more about the whole area.

- Given the best sensing algorithm for individual spectrums, the second problem is sensor scheduling. On one hand, more sensors assigned to a spectrum can produce better sensing results. On the other hand, sensors should be dispersed to different spectrums so that more spectrums can be sensed simultaneously.

- Communications within the SSN are also critical for the effectiveness of an SSN. A wired SSN is a simple but costly solution. By contrast, wireless SSN is attractive for its easy deployment and low-cost maintenance. SSN can use dedicated wireless resources such as the legacy ISM band or simply apply the DSA.

- Distributed design. An SSN with a central station to control is an easy design but will introduce additional overhead on communications, control, and energy cost. Localized sensing schemes that involve sensors in the vicinity are more attractive but challenging.

In the remainder of this article, we focus our attentions on the first problem, leaving others to future work.

\section{PROBLEM FORMULATION}

In SSNs, the very first problem is that, given a set of sensors assigned to a spectrum, which algorithm achieves the best sensing results? We call this the Sensing Error Minimization (SEM) problem. In this section, we first present the system model and the sensing model that we adopt, and then we formulate the problem and state the key challenges when addressing the problem.

\subsection{System Model}

Compared with the conventional spectrum sensing scheme, a unique requirement of SEM is that we care not only about the PU's presence at individual locations, but also about which areas are covered by the PUs. In SEM, we consider only one spectrum and assume that sensors have been well deployed. 
We model the spectrum as an "on/off" resource. Each spectrum is represented by its center frequency and constrained by the lower and upper cutoff frequencies. The spectrum resource is "on" when PUs are present at the location, and "off" otherwise. We assume a fully cooperative or controlled environment where sensors are willing to participate in the sensing tasks. SSNs under hostile or gaming environments are out of our discussions.

We assume that at each spectrum there is at most one PU. This is a reasonable assumption as in practice PUs have exclusive priority to use the spectrum. Different PUs will be far enough away to avoid interference between them. For instance, the coverage range of a Digital TV transmitter is $150.3 \mathrm{~km}$ [28]. To protect DTV users, different DTV transmitters, when they are using the same spectrum, must be several times this range apart. Compared with this distance, the size of the field in practice is fairly small. Accordingly, it will be very rare that one field covers two different PUs. When the field is big enough so that multiple PUs are present in the same spectrum, we can grid the field to an appropriate size (say, 150.3) so that one grid contains one PU only and then apply the single PU sensing algorithm. Since spectrum sensors are centrally controlled, sensors will cooperatively determine whether the single PU is present when they are scheduled to one spectrum at a time.

We assume sensors are aware of their precise locations by carrying GPS or applying localization algorithms [19]. Their locations are denoted as $L=\left\{\left(x_{i}, y_{i}\right), i=1, \ldots|V|\right\}$. Sensors will sense the PU's presence at their own locations. The application field is denoted as $A$.

\subsection{Sensing Model}

For sensing of individual sensors, we assume that energy detection [16] is applied, which consumes less energy and introduces short sensing times. Note that our method is generic for other sensing techniques by simple extension. The sensing results may be erroneous, and this is closely related to the context information (e.g., the distance between the PU and sensor).

Ideally, by the path-loss model, the Received Signal Strength (RSS) $P(d)$ in logarithm scale is $P(d)=P_{0}+10 k \log \left(\frac{d}{d_{0}}\right)$ where $k$ is the path-loss exponent, $d_{0}$ is a distance of reference location, $d$ is the distance between the primary transmitter, and the receiver and $P_{0}$ is a referencing transmitting power. Given a desired threshold $\lambda_{t h}$ of the Signal-Noise-Ratio for receivers, the contour of $P(d)=$ $\lambda_{t h}$ is the real boundary ${ }^{1}$ of PU's covered areas. In the ideal case, it is a circular arc.

In real environments, RSS is a random variable $x$ due to shadow fading and multi-path effects. Its probability density function can be modeled by Rayleigh fading model:

$$
\operatorname{Pr}(x)=\left\{\begin{array}{ll}
\frac{x}{\sigma^{2}} \exp \left(-\frac{x^{2}}{2 \sigma^{2}}\right), & x \geq 0 \\
0 & x<0
\end{array},\right.
$$

where $\sigma=\frac{P(d)}{\sqrt{\pi / 2}}$. Equation (1) implies that a lower $P(d)$ will have a smaller detection variance, and that a higher $P(d)$ results in a larger variance. For instance, supposing $\lambda_{t h}=-15 d B$, we have sensors of $P(d)=-15 d B$ declaring "off" with $P_{F A}=0.24$, and sensors of $P(d)=-10 d B$ "on" but have $P_{M D}=0.05$, and sensors of $P(d)=20 d B$ have $P_{M D}=0.01$. Figure 3 depicts the CDF of different $\mathrm{P}(\mathrm{d})$.

More measurements can reduce the error rate at the cost of more sensing time. When $m$ samples are used to make a single declaration, we have

$$
x=\sum_{i=1}^{m} x_{i}^{2} / m
$$

\footnotetext{
${ }^{1}$ In this article, we use the real boundary and the ground truth boundary interchangeably.
} 


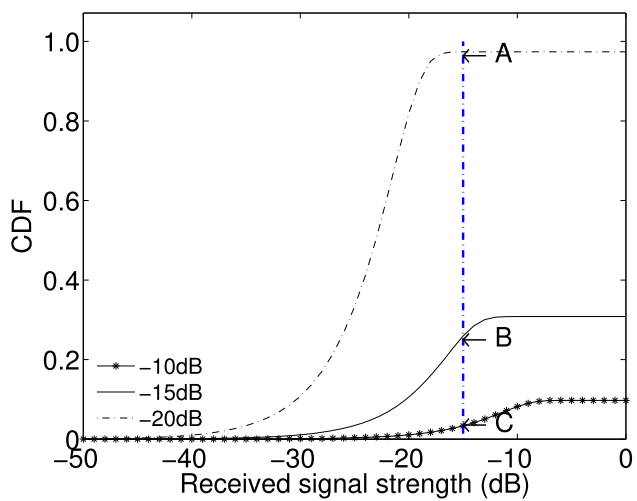

Fig. 3. The CDF of individual detected energy by Rayleigh fading model. The $x$-axis is the random variable to denote the detected energy level, and different curves represent those of different $P(d)$.

that follows the non chi-square distribution, where $x_{i}$ denotes the RSS of $i$ th measurement. By this scheme, the new $P_{M D}$ becomes

$$
\begin{aligned}
P_{M D}= & 1-e^{\frac{-\lambda}{2}} \sum_{k=0}^{m-2} \frac{1}{k !}\left(\frac{\lambda}{2}\right)^{k}+\left(\frac{1+P(d)}{P(d)}\right)^{m-1} \\
& \times\left(e^{\frac{-\lambda}{2(1+P(d))}}-e^{\frac{-\lambda}{2}} \sum_{k=0}^{m-2} \frac{1}{k !}\left(\frac{\lambda P(d)}{2(1+P(d))}\right)^{k}\right),
\end{aligned}
$$

where $\lambda=\sum_{i=1}^{k}\left(\frac{\mu_{i}}{\sigma_{i}}\right)^{2}$ and $\mu$ is the means of the measurements.

Definition 3.1. Let $h(l)$ denote a declaration of a sensor at location $l=(x, y)$ where $h(l)=0$ when the average of $m$ sensing samples is above a given threshold, implying that no PUs exist, and $h(l)=1$ when otherwise. The declaration $h(l)$ is also written as $h\left(v_{i}\right), v_{i} \in V$ if $v_{i}$ is at $l$.

Definition 3.2. We define declaration set $H(L)$ as the set of declarations at all sensor locations in $L, H(L)=\{h(l), l \in L\}$. We also write $H(L)$ as $H(V)$ if $V$ 's location set is $L$.

We use $\overline{\mathrm{H}}(L)=\{\bar{h}(l), l \in L\}$ to denote the ground truth of the presence of PUs.

Definition 3.3. Boundary in a field $A$ is a real-valued function $f: R^{2} \rightarrow R$. A location $l=\{x, y\} \in$ $A$ is assigned to the "off" class (no PU presence) if $f(l) \geq 0$ and to the "on" class if otherwise. The declaration set $H_{f}(A)$ of $f$ can be computed by

$$
\begin{aligned}
H_{f}(A)=\left\{h_{f}(l) \mid l \in A,\right. & (f(l) \geq 0) \cap\left(h_{f}(l)=0\right), \\
& \left.(f(l)<0) \cap\left(h_{f}(l)=1\right)\right\}
\end{aligned}
$$

\subsection{Illustrative Example}

Figure 4 gives an illustration example of SEM. In this example, there is one PU (primary transmitter) and a real boundary (blue curve) between the PU-covered and -uncovered areas. Sensors can sense the spectrum and make declarations based on their sensing results. The solid dots represent "on" sensors (i.e., PUs exist) and circle dots are "off." As the sensing can be erroneous due to hardware constraints, both circles on the left and solids on the right of the real boundary are erroneous. We, however, have no knowledge of which sensors are correct. The basic goal of spectrum sensing in SSN is to accurately detect this real boundary based on the erroneous inputs. 


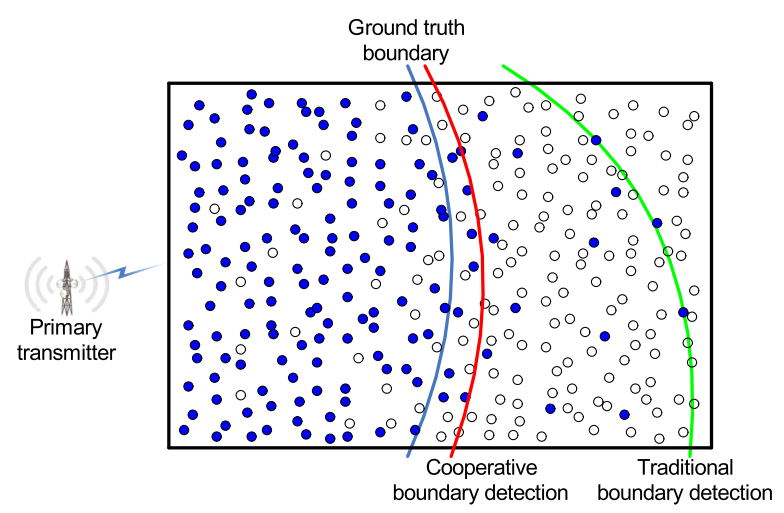

Fig. 4. An illustration of the system model and the motivation example. The blue curve is the ground truth boundary; the green one is obtained by traditional boundary detection and the red one is detected by cooperative boundary detection.

The boundary detection in SSN is inherently different from traditional boundary detection [36], which is mostly based on correct inputs. In the example of Figure 4, the best that the traditional approach can do is to draw a convex envelop (green curve) to enclose all the "on" nodes. As the erroneous declarations are notable, the gap between green and blue curves is large.

\subsection{Problem Statement and Key Challenges}

The problem can be formulated as follows.

Sensing Error Minimization (SEM): Given a set of spectrum sensors $V$, their locations $L$ in $A$, and the declaration set $H(L)$, the problem is to find a boundary function $f$ with the minimal detection error; that is,

$$
\text { minimize : } \bar{H}(A) \oplus H_{f}(A)=\sum_{l \in A}\left|\bar{h}(l) \oplus h_{f}(l)\right|,
$$

where $H_{f}(A)$ is $f$ 's declaration set and $\oplus$ is the XOR binary operation. Here, the cap means the ground truth.

Because of the nature of the erroneous input, no algorithm can be guaranteed correct. Consider a simple extreme case in which all the declarations are the same: say, "on." In such a case, no boundary can be drawn even if we are given the knowledge that there is a boundary. In general, there are several key challenges when we explore solutions.

The first challenge is that the sensing errors are not sensitive to distance. A node far from the real boundary also has a high probability of error. Thus, the correct and erroneous declarations will be mixed together. To tackle this challenge, we intelligently leverage the cooperative spectrum sensing concept. The basic idea is that a sensor is very likely to have an erroneous declaration if all its neighbor sensors in proximity have the opposite declaration. The key issue is how to define the neighbors of a sensor, how to deal with the multiple errors, and how to determine its effectiveness.

The second challenge is the lack of extra tools for verification. After the declaration calibration, there may still be many erroneous declarations, yet we have no means to determine which are correct and which are wrong. For this challenge, we note that such erroneous declarations, if there are any, will be very likely to be distributed near the real boundary. Accordingly, we design an error-tolerance boundary derivation algorithm and carefully set the penalty for an erroneous declaration. As such, the erroneous declaration can be effectively mitigated. 


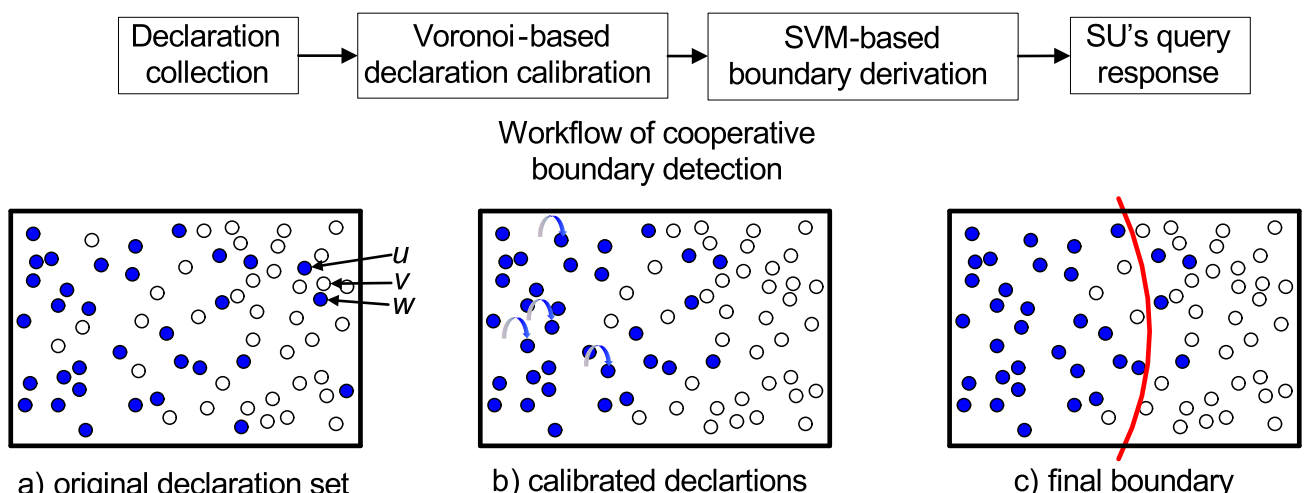

Fig. 5. Workflow of cooperative boundary detection. (a) The original declaration set obtained from the sensors, (b) the declarations after the reciprocal declaration calibration, and (c) the final boundary derived by error-tolerance boundary derivation.

The third major challenge is that the ground truth boundary can be any arbitrary shape due to the fading effect. For this challenge, we have the following observations. In the ideal case of a path-loss model, the real boundary is a circular arc. It becomes irregular because of shadow fading and multi-path effects. According to the IEEE 802.22 standard, however, spectrum opportunities coming from shadow fading and multi-path effects are not allowed access. More specifically, in IEEE 802.22, misdetections include those incurred by shadow fading and multi-path effect. In other words, a vacant spectrum at a location caused by shadow fading and multi-path is counted as misdetection but not as a spectrum opportunity. The corresponding area will be considered as "PU present." From the SU's point of view, it makes no difference whether the spectrum exists but cannot be used or the opportunity does not exist at all. This makes the design of spectrum sensing easier. We can simply assume that the target boundary has the regular shape of a circular arc.

According to these design principles, we develop cooperative boundary detection, which will be detailed in the next section.

\section{COOPERATIVE BOUNDARY DETECTION}

In this section, we introduce the design of the cooperative boundary detection algorithm. We first describe the algorithm architecture and then provide the detailed design of each components.

\subsection{Design Overview}

Cooperative boundary detection is a two-stage scheme. It first runs a calibration algorithm, trying to reduce input errors. After calibration, the erroneous declarations can be effectively reduced and the remaining erroneous ones, if there are any, will be likely to be deployed near the real boundary. Taking the calibrated declarations as the new input, in the second stage, an error-tolerance boundary derivation algorithm is invoked to depict the boundary. The derivation algorithm is based on recent advances in Support Vector Machines (SVM).

Figure 5 depicts the general architecture of cooperative boundary detection. Initially, the server should collect declarations from all sensors in the field. It then constructs declaration set $H(V)$ as the original input (Figure 5(a)). Based on $H(V)$, the server executes the reciprocal declaration calibration algorithm and obtains a calibrated declaration set $H_{c}(V)$ (Figure 5(b) with the transferred labels). The calibrated declaration set $H_{c}(V)$ will then be used as the new input of the errortolerance boundary derivation to compute the boundary function (Figure 5(c)). In the next section, we introduce these two components in details. 


\subsection{Reciprocal Declaration Calibration}

4.2.1 Design Principles. Declaration calibration is based on a simple idea. A pair of nearby sensors with the same declarations can mutually "support" each other. Otherwise, two opposite declarations imply that either the ground truth boundary is in between, or one of the two declarations is incorrect and should be flipped. Note that it is still possible that two same nearby declarations are both errors while their confidence of correctness is dramatically increased. The key issue is the definition of "neighborhood."

A naive approach to define neighborhood is range-based. It is also referred to as a disk model, in that each node has a deterministic range. All other nodes within the range are considered neighbors of this node. The disk model is, however, not appropriate because, in spectrum sensing, there is no sensing range and declarations only represent the sensing results of individual locations. Moreover, directions are more important than distance. In the example of Figure 5, sensors $u$ and $w$ are very close to each other and have the same declarations. However, they should not support each other as $u$ has been surrounded by opposite declarations.

Inspired by these observations, we propose a Voronoi-based declaration calibration algorithm. A Voronoi diagram [7] and its dual form Delaunary Triangulation (DT) are powerful tools that have been widely utilized. Due to space limitations, we omit the details; interested readers may refer de Berg et al. [7].

Definition 4.1. Define $\Upsilon\left(v_{i}\right)$, the Voronoi cell for a sensor $v_{i}$, to be the set of points in $A$ that are closer to $v_{i}$ than any other sensor; that is,

$$
\Upsilon\left(v_{i}\right)=\left\{p \mid d_{p, v_{i}} \leq d_{p, v_{j}}, p \in A, i \neq j\right\},
$$

where $d_{p, v}$ is the Euclidean distance between $p$ and $v$.

Definition 4.2. Two sensors $u$ and $v$ are Voronoi neighbors if $\Upsilon(u)$ and $\Upsilon(v)$ share a common edge in the Voronoi diagram. The set of all Voronoi neighbors of a given sensor $v_{i}$ is denoted as $N\left(v_{i}\right)$. Voronoi neighbors are also the neighbors in DT.

Definition 4.3. Given a sensor $v$ and a declaration set $H(V)$, another sensor $u$ is a "supporter" of $v$ if $h(u)=h(v)$ and an "objector" if otherwise. Let $N_{s}^{H}(v)$ denote the supporter set and $N_{o}^{H}\left(v_{i}\right)$ the objector set; that is,

$$
\begin{aligned}
& N_{s}^{H}(v)=\left\{u \mid h(u) \oplus h\left(v_{i}\right)=0, u \in V\right\} \\
& N_{o}^{H}(v)=\left\{u \mid h(u) \oplus h\left(v_{i}\right)=1, u \in V\right\} .
\end{aligned}
$$

Noting that a neighbor is either a support or an objector, we have

$$
\begin{aligned}
& N_{o}^{H}(v) \cup N_{s}^{H}(v)=V \\
& N_{o}^{H}(v) \cap N_{s}^{H}(v)=\Phi .
\end{aligned}
$$

Definition 4.4. A sensor $v_{i}$ is safe if at least one of the three following conditions is satisfied:

(1) All $v_{i}$ 's Voronoi neighbors are $v_{i}$ 's support, or;

(2) $v_{i}$ is along the perimeters of the field, or;

(3) There exists a path in the DT from $v_{i}$ to a $v_{i}$ 's safe supporter such that all nodes along the path are also $v_{i}$ 's supporters.

In our reciprocal declaration calibration, all the safe nodes are kept unchanged while the remaining unsafe sensors should flip their declarations until safe. Later, we will prove that, after calibration, all sensors will be safe with respect to the calibrated declaration set. Figure 6 gives the pseudo-code of the reciprocal declaration calibration algorithm. 


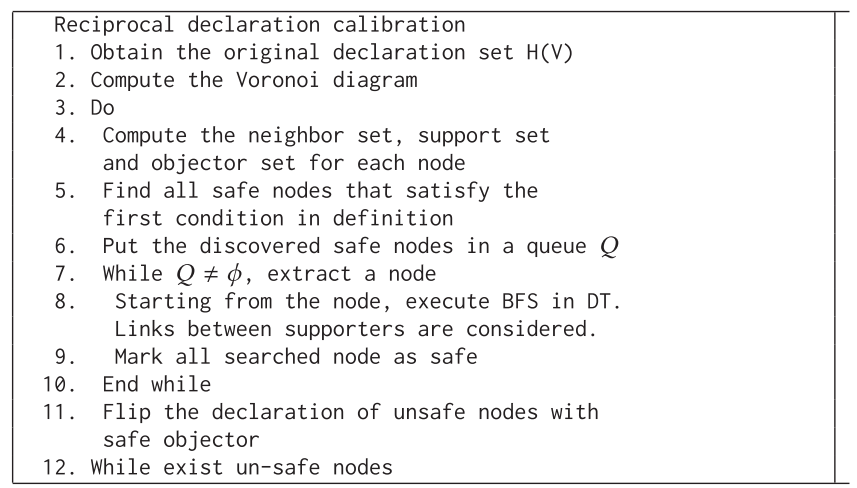

Fig. 6. Pseudo-code of the reciprocal declaration calibration.

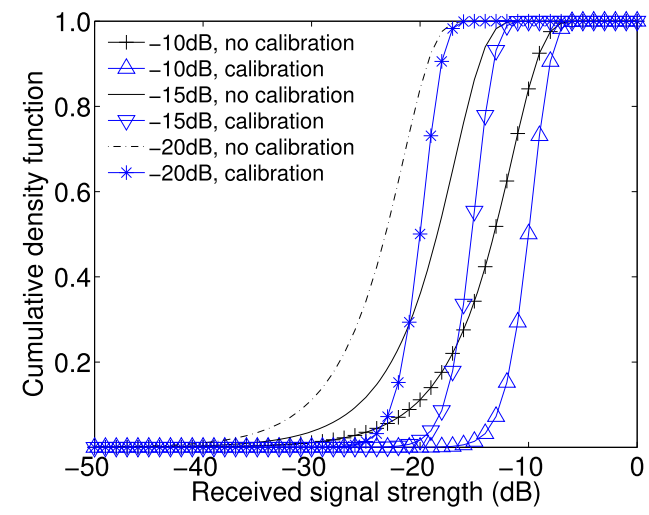

Fig. 7. The sensing performance before and after the reciprocal declaration calibration.

\subsubsection{Analytic Results.}

THEOREM 4.5. Given a set of sensors $V$ and the calibrated declaration set $H_{c}$, the sensing performance of a safe node with $N$ connected supporters is

$$
P_{M D}\left(h_{c}\right)=P_{M D}^{N}
$$

and

$$
P_{F A}\left(h_{c}\right)=1-\left(1-P_{F A}\right)^{N},
$$

where $h_{c} \in H_{c}$ is the node's calibrated declaration under the assumption that every sensor has the same $P_{M D}$ and $P_{F A}$ for a given signal strength. Note that $N \geq 4$ in practice.

Proof. According to the properties of Voronoi diagrams, each node has at least three neighbors. By the definition of "safe," all the neighbors are supporters, and, therefore, in the vicinity of the node, there are four nodes having the same declarations (include the node itself). We thus have $N \geq 4$. Each node has the $P_{M D}$ and $P_{F A}$. By inclusion-exclusion principles, we have the equations.

Figure 7 shows some sensing performance improvement after calibration. Assuming the RSS threshold is $-15 d B$, for sensors of $P(d)=-15 d B, P_{M D}$ is reduced from 0.24 to 0.02 , and for sensors of $P(d)=-10 d B, P_{M D}$ is reduced from 0.05 to 0.00001 . Note that we set $N=4$ conservatively; in practice, $N$ is much larger. 


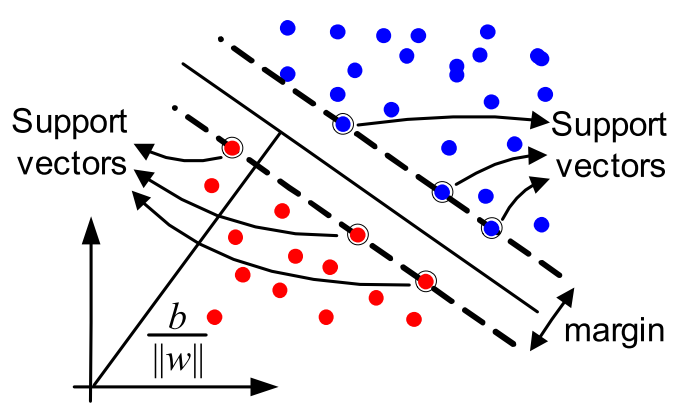

Fig. 8. An illustration of a Support Vector Machine.

The time for declaration set collection mainly depends on the communication protocols between the sensors and the server. The best know algorithm for a Voronoi diagram is $O(n \log n)$. The computation of the neighbor set depends on the number of links in DT. Recall that DT is a planar graph, and the total number of links is no more than $3 n-6$. The computation cost for Step 4 is $O(n)$. We separate the Step 4 and Step 5 for simplicity of presentation, while in practice these two steps can be run concurrently without additional overhead. Line 7 is another important step. As each node can be set as safe at most once, the total cost of Step 9 is no more than $n$. Step 11 guarantees that every node flips at most once. Summing all these costs, we conclude that the computation cost of reciprocal declaration calibration is $O(n \log n)$, which is quite efficient. The most important thing is that DT has at most $3 n-6$ links as DT is a planar graph. In DT, the average node degree is a constant 3.

\subsection{Error-Tolerance Boundary Derivation}

In this part, we present the bondary derivation algorithm. We first present the challenges we are facing and then give a brief introduction to the SVM. In the last subsection, we present our design.

4.3.1 Challenges. To draw an accurate boundary, we face a number of challenges.

The first challenge is the infinite number of candidate boundary functions. Given the input declaration set, assuming all declarations are correct, there are an infinite number of boundaries that can produce the same declaration set as the input. In other words, any of them can be the solution. We need to choose the most adequate one with the fewest errors. The intuition is that the boundary with the maximal margin width between the two declaration classes is the correct one.

The second challenge is that the input declaration set is not error-free. Calibration can effectively reduce the erroneous declarations but can provide no error-free guarantees. Nevertheless, we are given the information that the erroneous declarations should be distributed near the real boundary. In other words, the boundary producing minimal errors on the calibrated declarations will be the one closest to the real boundary.

4.3.2 Support Vector Machines. Based on these two observations, we select an SVM as the fundamental tool to derive the boundary function.

An SVM in general is a linear classifier that classifies the space by a linear function. Consider the simple illustration of a 2D feature space in Figure 8. Suppose we have a number of labeled nodes (blue and red) and want to find a classifier to group these nodes. Though all lines between the two group can be the classifier, intuitively the one with the largest margin should perform the best for other nonlabeled nodes. Mathematically, this classifier can be found by finding the largest number 
of support vectors, as illustrated in the figure. An SVM can also produce nonlinear classifiers by mathematical tricks. This trick will be applied in our work.

Formally speaking, binary classification is performed by using a real-valued linear function $f: R^{k} \rightarrow R$ where $k$ is the dimension of the input space. In our application scenarios, $K=2$ and $R^{k}=A$. For a target location $l=\{x, y\} \in A$, it is assigned to the "off" state if $f(l) \geq 0$, meaning that the location has no PUs, and to "on" if otherwise. The linear function $f(l)$ can be expressed as

$$
f(l)=\langle w \cdot l\rangle+b,
$$

where $w=\left[w_{x}, w_{y}\right]$ represents the direction perpendicular to $f(l), w \cdot l=w_{x} \cdot x+w_{y} \cdot y$ is the inner dot product, and $b$ moves the linear classifier parallel to itself (for lines in 2-D space, $b$ is the intercept and $w$ is the slope of the line). When all the labels (declarations) are correct, the linear classification function $f$ is optimal when it satisfies the following conditions.

$$
\begin{array}{ll}
\min : & \langle w \cdot w\rangle+C \cdot \sum_{i=1}^{n} \varepsilon_{i} \\
\text { s.t. } & : \quad\left\langle w \cdot l_{i}\right\rangle+b \geq 0, i \in[1, n], h_{i}=0 \\
& \left\langle w \cdot l_{i}\right\rangle+b<0, i \in[1, n], h_{i}=1,
\end{array}
$$

where $\langle w \cdot w\rangle^{2}$ is the margin width, and the cost parameter $C$ strikes a tradeoff between the margin width and the tolerance of the input errors. When all labels (declarations) are correct, the cost parameter $C$ is 0 , and the optimal solution of function $f$ can be found by quadratic programming [23].

4.3.3 Dealing with Arbitrary Boundary Shape. An SVM can only derive linear boundary functions. As the target boundary function is a circular arc but not linear, we need to change the representation of the sample data from the 2-D input space to a higher dimensional feature space. As such, the target boundary function becomes linear; that is,

$$
l=(x, y) \in A \mapsto \varphi(l)=\left(\varphi_{1}(l), \varphi_{2}(l), \ldots \varphi_{K}(l)\right) \in R^{K} .
$$

Next, we will show how to obtain $\varphi_{i}(l)$ and $R^{K}$.

To derive the feature function $\varphi$, we write the target boundary function as

$$
f:\left(x-x_{0}\right)^{2}+\left(y-y_{0}\right)^{2}=R_{t h}^{2},
$$

where $\left(x_{0}, y_{0}\right)$ is the location of the primary transmitter and $R_{t h}$ is the keep-out radius of the primary transmitter. The key issue in boundary detection then becomes finding $\left(x_{0}, y_{0}\right)$ and $R_{t h}$ of minimal detection errors. Equation (2) can be rewritten as

$$
f:<w \cdot \varphi(l)>+b=0
$$

where

$$
\begin{aligned}
w & =\left[1,-2 x_{0},-2 y_{0}\right] \\
\varphi(l) & =\left[x^{2}+y^{2}, x, y\right] \\
b & =x_{0}^{2}+y_{0}^{2}-R_{t h}^{2} .
\end{aligned}
$$

In other words, in a 3-D feature space, the target boundary function is a linear function (i.e., $\mathrm{K}=3$ for $\left.R^{K}\right)$. The mapping function for the feature space is

$$
\forall l=(x, y) \in R^{2}, \varphi(l)=\left[\varphi_{1}(l), \ldots, \varphi_{3}(l)\right] \in R^{3},
$$

where $\varphi_{1}(l)=x^{2}+y^{2}, \varphi_{2}(l)=x$, and $\varphi_{4}(l)=y$.

\footnotetext{
${ }^{2}$ Indeed, the margin width should be $\frac{2}{\sqrt{\langle\boldsymbol{w} \cdot \boldsymbol{w}\rangle}}$. It is equivalent to $\langle w \cdot w\rangle$ by an appropriate scalar.
} 


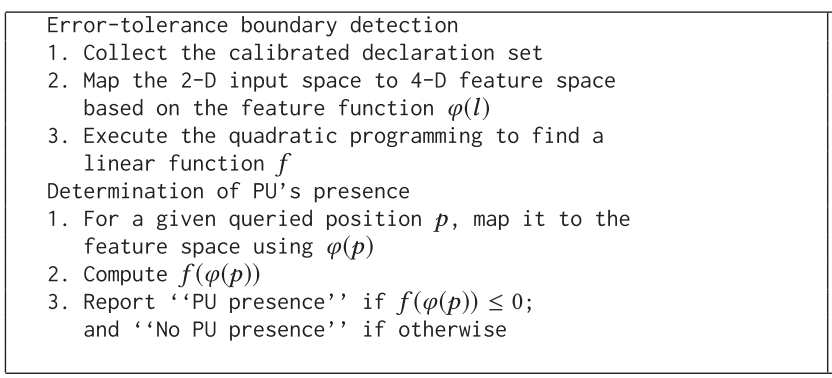

Fig. 9. Pseudocode of the error-tolerance boundary derivation and the determination of PU presence for a location $p$.

A last question when applying an SVM is the erroneous inputs. This can be addressed by setting an appropriate cost parameter to the erroneous declarations. Combining all these, we design an SVM with the target boundary function $f$ such that it can

$$
\begin{array}{cl}
\text { minimize } & : \quad\langle w \cdot w\rangle+C \cdot \sum_{i=1}^{n} \varepsilon_{i} \\
\text { subject to }: & \left\langle w \cdot l_{i}\right\rangle+b \geq 0, i \in[1, n], h_{i}=0 \\
& \left\langle w \cdot l_{i}\right\rangle+b<0, i \in[1, n], h_{i}=1,
\end{array}
$$

where $\langle w \cdot w\rangle$ is the margin width between different classes (sensors with different declarations) and the cost parameter $C$ that helps to find the tradeoff between the maximization of margin width and the penalty of erroneous declarations. When all labels (declarations) are correct, there is no erroneous input, and the cost parameter $C$ is 0 . When the erroneous declarations are many, a higher $C$ is needed. In practice, we study the impact of the parameter $C$ through experiments. The optimal solution function $f$ in Equation (3) can be found by quadratic programming [23].

Figure 9 gives the algorithm pseudo-code.

4.3.4 Analytic Results. The complexity of the computation algorithm is straightforward. The mapping from 2-D input space to the 3-D feature space requires $O(n)$ time. The complexity of computing the linear classifier $f$ depends on the efficiency of the quadratic programming, which can be solved in polynomial time by the state-of-art algorithms [23]. Therefore, the overall algorithm complexity is polynomial.

THEOREM 4.6. The boundary computed by the error-tolerance boundary derivation is asymptotically the real boundary, provided all the sensors have the correct declarations and the real boundary is a circular arc.

Proof. We prove this theorem by showing that (i) the output linear function $\mathrm{f}$ in the 3 - $\mathrm{D}$ feature space defines a circular arc in 2-D input space and (ii) the difference between this arc and the real boundary approaches 0 as the number of sensors goes to infinity and the distances between Voronoi neighbors approaches 0 .

For the first claim, the output boundary obtained by the error-tolerance boundary is a linear function in the 3-D feature space, $f:\langle w \cdot X\rangle+b=0$ where $w=\left[w_{1}, w_{2}, w_{3}\right] \in R^{3}$ and $X=\left[x_{1}, x_{2}, x_{3}\right] \in R^{3}$. In particular, is the $w=\left[w_{1}, w_{2}, w_{3}\right] \in R^{3}$ is the perpendicular vector of the plane. Let $x_{0}=-\frac{w_{2}}{w_{1}}, y_{0}=-\frac{w_{3}}{w_{1}}$ and $R=\sqrt{b-\left(\frac{w_{2}}{w_{1}}\right)^{2}-\left(\frac{w_{2}}{w_{1}}\right)^{2}}$. We have that any points in the arc $f^{\prime}:\left(x-x_{0}\right)^{2}+\left(y-y_{0}\right)^{2}=R^{2}$ will be located on the plane defined by $\mathrm{f}$. 
For the second claim, since we are under the assumption that all sensors have the correct declarations, the control parameter $C$ will be set at 0 . Therefore, the minimization function in quadratic programming is $<w . w\rangle$, which is the margin width between the two classes of samples. The margin width is two times the upper bound of the difference between the derived boundary and the real boundary in the feature space. As the distances between Voronoi neighbors approaches 0 , the margin width approaches 0 as well. As a result, the difference between the derived boundary and the real boundary also approaches 0 . In other words, the derived boundary is an asymptote of the real boundary.

\subsection{Discussion}

In practice, there may be multiple spectrums to be sensed and accessed. Supposing there are a number of, say $N$, spectrum sensors and $T$ primary spectrums, and we need to determine which sensors are assigned to sense each spectrum in order to maximize the dynamic spectrum access opportunities. On one hand, more sensors in a spectrum can sense more accurately. On the other hand, sensors should be assigned to different spectrums so that more spectrums can be sensed simultaneously. Let $n_{k}$ denote the number of sensors assigned in the $k$ th spectrum. Clearly, we have

$$
n=\sum_{k=1}^{t} n_{k}, t<T,
$$

where $t$ is the total number of simultaneously sensed spectrums.

Definition 4.7. Given a sensed spectrum, a sensing result is a boundary function $f_{S}: R^{2} \rightarrow\{0,1\}$ such that a location $l=\{x, y\} \in A$ is sensed as an "on" status if $f_{S}(l) \geq 0$ or "off" status if otherwise.

For the $k$ th sensed channel, this sensing result is called the $k$ th sensed boundary $f_{S}^{k}$. The real boundary, which can also be expressed as a boundary function $f_{R}^{k}(l): R^{2} \rightarrow\{0,1\}$ with a similar definition is called the kth real boundary or real boundary.

Definition 4.8. Given a sensed boundary $f_{S}$ and a real boundary $f_{R}$, the area is partitioned into four subareas:

(1) Sensed/whitespace area $L_{R} / L_{S}$ are the PU-covered/PU-uncovered areas that are correctly sensed by the sensed boundaries; that is,

$$
\begin{aligned}
& L_{R}=\left\{l \mid l \in A, f_{R}(l) \geq 0 \cap f_{S}(l) \geq 0\right\} \\
& L_{S}=\left\{l \mid l \in A, f_{S}(l)<0 \cap f_{S}(l)<0\right\}
\end{aligned}
$$

(2) Misdetection area $L_{M D}$ is the area covered by PUs but sensed as uncovered,

$$
L_{M D}=\left\{l \mid l \in A, f_{R}(l) \geq 0 \cap f_{S}(l)<0\right\}
$$

(3) False-alarm area $L_{F A}$ is the area uncovered by PUs but sensed as covered; that is,

$$
L_{F A}=\left\{l \mid l \in A, f_{R}(l)<0 \cap f_{S}(l) \geq 0\right\}
$$

An example of the four subareas is depicted in Figure 10. Note that $L_{R}$ will be well protected and $L_{S}$ will be adequately utilized for SU spectrum access. In $L_{M D}$, PUs will be potentially harmed, and, in $L_{F A}$, the SU spectrum opportunity is wasted. Therefore, smaller $L_{M D}$ and $L_{F A}$ are preferred, while these two goals are naturally contradicted [16].

The whitespace area $L_{S}$ can be used to assess the sensing effectiveness of a single spectrum and to evaluate the effectiveness of the channel assignment algorithm. This assessment is, however, largely dependent on the size of the real whitespace areas (i.e., $L_{S}+L_{F A}$ ). To cancel these application-dependent effects, we use a normalized value. In addition, we have mandatory requirements on the sensing accuracy (e.g., in the IEEE 802.22 standard, both the misdetection 


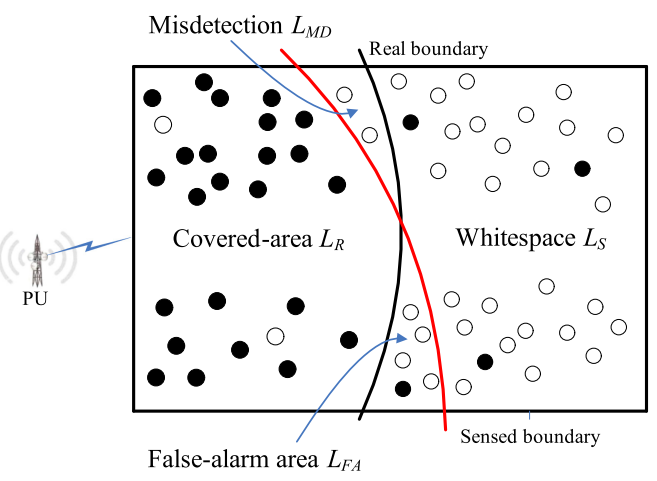

Fig. 10. An example of the sensing results. The sensed and real boundaries as well as the four subareas are illustrated.

probability $P_{M D}$ and the false alarm probability $P_{F A}$ must be no more than 0.1 ). A sensed spectrum failing to satisfy these requirements cannot be used by SUs and thus provides no effectiveness.

Accordingly, assigning spectrum sensors to one spectrum will have the utility based on the union of $L_{R}$ and $L_{S}$, while $L_{M D}$ and $L_{F A}$ have no utility. The total utility of all the spectrum is the sum of the utility of each spectrum. It is easy to see that this maximization problem is a variation of the $0-1$ knapsack problem, which is NP-complete. In a preliminary work, we design some heuristic algorithms [3], and more advanced designs are left for future work.

\section{PERFORMANCE EVALUATIONS}

In this section, we study the impact of the control parameters and evaluate their performance. We design and implement a prototype spectrum sensing system using a 2.4G ISM band to emulate a licensed band. We also conduct comprehensive simulations to evaluate performance under different settings. Recall that the error can be calculated as

$$
\bar{H}(A) \oplus H_{c}(A)=\sum_{l \in A}\left|\bar{h}(l) \oplus h_{c}(l)\right|,
$$

which will be used as our evaluation metric. As the shape of the real boundary is not regular and can be arbitrary, we apply the Monte-Carlo method to measure this error. The studied parameters include the field size, the density of sensor deployment, and the sensor distribution. Each measurement is obtained by averaging 100 independent runs.

We compare cooperative boundary detection with three alternatives.

- The first is the traditional algorithm with the simple OR-rule [12] for data fusion [36]. We term this one "Traditional."

- The second alternative is the simple SVM on the raw data without our reciprocal calibration; we call this "Simple SVM."

- The last one is again an SVM, but assume all sensors have the correct declarations. This is the best we can do to draw the boundary and is depicted as "Upper Bound."

- Our proposed scheme is termed "Cooperative."

\subsection{Simulation Results}

Here, we present simulation results. We adopt the Rayleigh model and follow the IEEE 802.22 standard [27] to simulate the sensing behavior of individual sensors. Two distributions of sensors are investigated. One is a random deployment following Poisson distribution, and the other is a 


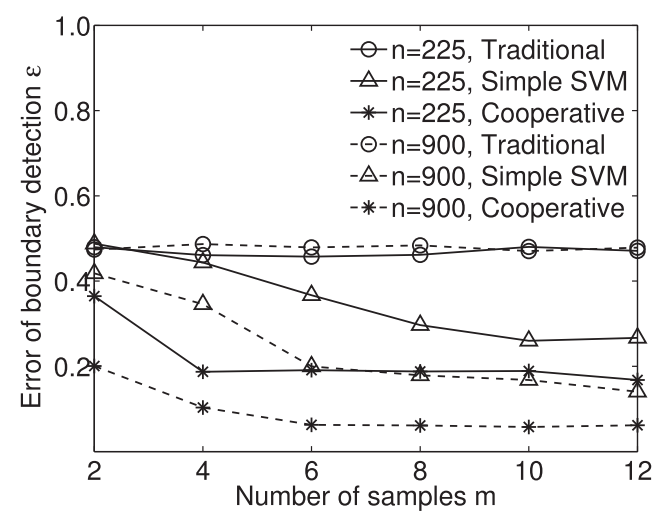

Fig. 11. Impact of the number of samples $m$ when signaling spectrums.

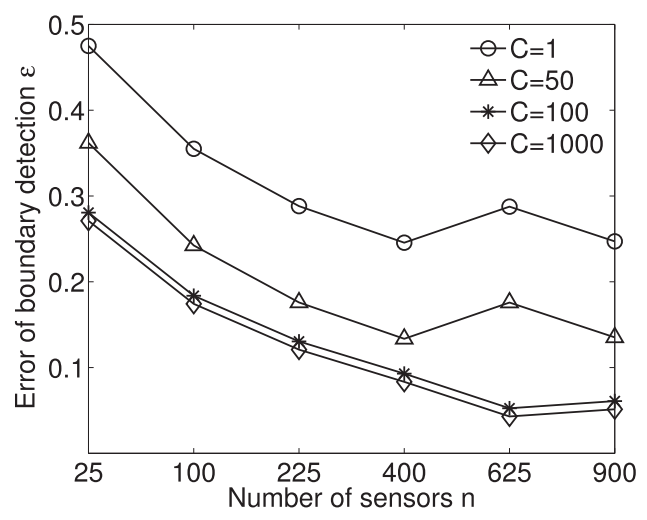

Fig. 12. Impact of the cost parameter $C$ in errortolerance boundary derivation.

uniformly random distribution in which the field is partitioned into fixed size grids. The number of deployed sensors varies from 25 to 900 . We also evaluate the performance of different algorithms under different field sizes. According to the experimental setting, $15 \mathrm{~km}$ corresponding to $1 \mathrm{~dB}$ radio attenuation of PUs.

5.1.1 Impact of Number of Samples $m$. Figure 11 shows the impact of $m$ the number of samples when individual sensors sense the spectrum. The field size is set to $15 \times 15 \mathrm{~km}^{2}$. Two numbers of sensors are deployed to the field: 225 and 900 . We apply uniformly random deployment, and thus in each side there are about 15 nodes.

From the results, we find that the traditional boundary detections can get little benefit from more signaling samples. The error is steadily around $50 \%$, regardless of the number of deployed sensors and $m$. This is mainly because the accuracy of the traditional approach heavily relies on those sensors with "on" declarations. As the OR-rule is applied in data fusion, three "on" sensors are sufficient to draw the boundary. As a result, reducing the erroneous declarations does not help much. Compared with this naive approach, a simple SVM gets more benefits. In a simple SVM, one more declaration may move the boundary in the correct direction, and thus fewer errors produce higher accuracy. In our experiments, $m=6$ can reduce the error from $42 \%$ to $20 \%$ compared with $m=2$. Cooperative boundary detection is able to obtain a satisfactory result even when $m$ is relatively low. This is mainly due to the power of the reciprocal declaration calibrations, which can greatly reduce error declarations. Because many errors can be calibrated, cooperative boundary detection provides a similar performance with a small $m$ as the simple SVM with a large $m$.

5.1.2 Impact of Cost Parameter $C$. In this set of experiments, we study the cost parameter $C$ in Equation (7). The cost parameter $C$ strikes a tradeoff between the detection error and the number of error declarations. Ideally, a higher density leads to a smaller detection error but more declaration errors, and therefore a larger $C$ is preferable. Figure 12 depicts the error of cooperative boundary detection against the real boundary. We adopt uniform random deployment, and the numbers of sensors $n$ varies from 25 to 900 . The field size is fixed as $15 \mathrm{~km}^{2}$. In general, a larger cost parameter $C$ can really improve the accuracy as the error declarations will obtain more penalties. As $C$ is more than 100, the penalties are sufficient to compensate the effect of error declarations and thus the improvement of further increasing $C$ becomes minor. In practice, we suggest $C>100$. As more nodes are deployed, a larger $C$ is needed. 


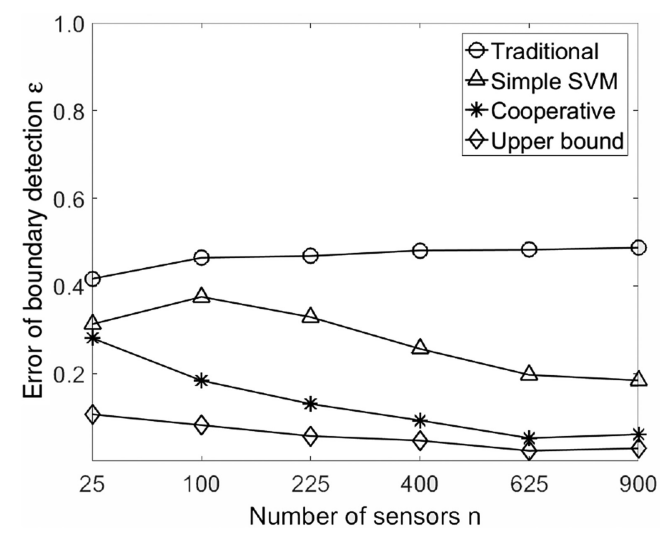

Fig. 13. Scalability of different approaches with different number $n$ of nodes.

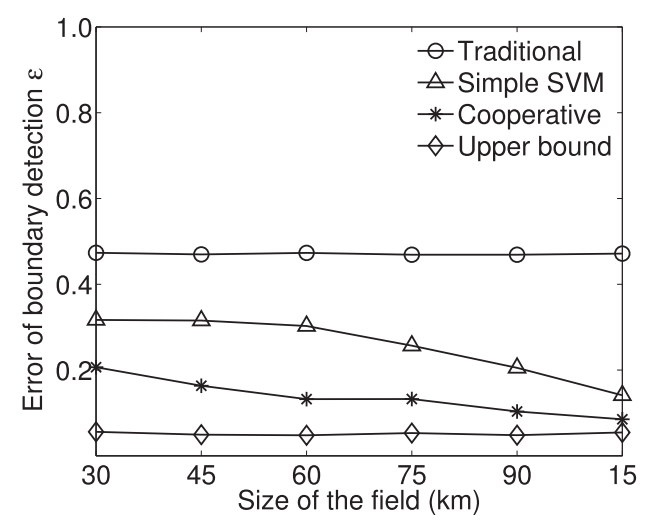

Fig. 14. Errors of boundary detections in settings of different field sizes.

5.1.3 Scalability of Different Approaches. In this set of experiment, we study the scalability of different approaches on performance. The field size is fixed as $1 \mathrm{~km}^{2}$ and the number of samples $m$ is set to 1. Four alternatives are studied; namely, traditional boundary detection, the simple SVM, our proposed cooperative boundary detection, and the upper bound under ideal cases. Figure 13 plots the errors of these four approaches with $n$ from 25 to 900 . The traditional approach has a slight performance degradation as $n$ scales up. From $n=25$ to $n=900$, its error increases from $42 \%$ to $49 \%$. Regarding the upper bound error $4 \%$, the relative error is about $45 \%(n=900)$. For the simple SVM, as $n$ scales up, the error declarations are more likely to be overcome by correct ones, and thus the error steadily decreases. From $n=100$ to $n=900$, it decreases from $38 \%$ to $18 \%$. We, however, are not clear why its performance becomes worse when the network scales from 25 to 100 , which will need further investigations. For cooperative boundary detection, by the power of reciprocal declaration calibration, the improvement is significant. When $n$ is small, the calibration is limited as the correct declarations are not sufficient to dominate incorrect ones. As more sensors are deployed, the corrected ones become the majority. As a result, cooperative boundary detection significantly reduces the error from $28 \%$ of $n=25$ to $7 \%$ of $n=900$. Compared with the upper bound of $4 \%$, the relative error is only $3 \%$. In other words, the detection error is reduced by $95 \%$, from $45 \%$ to $3 \%$, and the average reduction is around $85 \%$, from $45 \%$ to $7 \%$.

5.1.4 Impact of Field Size. Figure 14 shows the errors of different approaches under different field sizes. We set $n=400$, while the length of the field ranges from $15 \mathrm{~km}$ to $90 \mathrm{~km}$. In general, as the field size increases, sensors in different areas will have more differences in the probabilities of erroneous declarations. The traditional boundary detection can hardly leverage this features as its performance only depends on three false alarm nodes. Both simple SVM and the cooperative boundary detection can benefit from these features, while the cooperative boundary detection can take more advantage.

5.1.5 Impact of Different Distributions. Figure 15 plots the performance under different distributions. Compared with those in Figure 13 of uniform distribution, simple SVM and cooperative boundary detection have similar results, implying that these two algorithms are robust to nonuniform distributions. The traditional approach has a slight performance degradation. For the upper bound, notice that under nonuniform distribution there are more variances between nearby sensor distances. As upper bound is closely related to the nearby sensor distance, in a low network scale setup, the error of the upper bound is larger than that in Figure 13. 


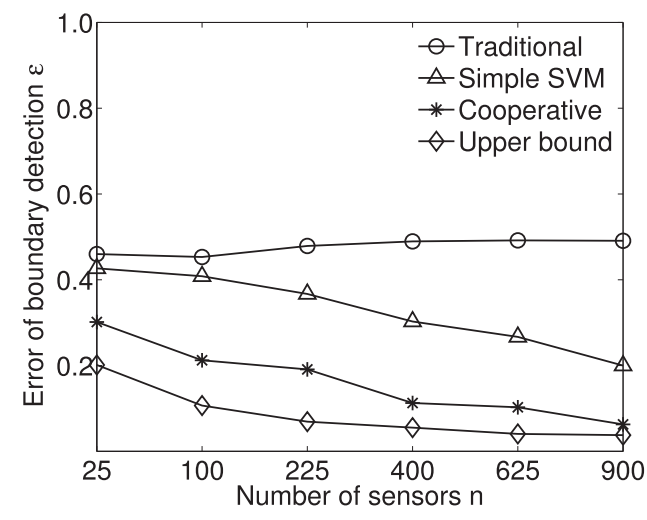

Fig. 15. Scalability of approaches under Poisson distribution.

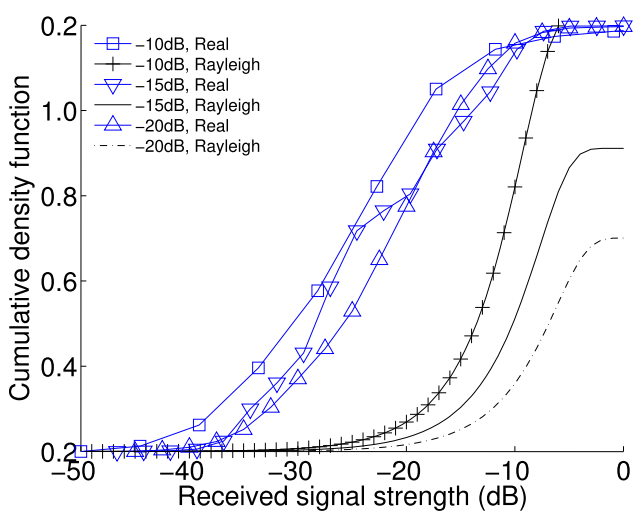

Fig. 16. Field measurements of signaling spectrum comparing with Rayleigh Model.

\subsection{Impacts of the Key Factors}

In this subsection, we investigate the impacts of the sensor deployment and the cardinality of the sensor set on sensing effectiveness through simulation experiments.

In our simulations, we use our previous work [39] as the spectrum sensing algorithm. We adopt the Rayleigh model to model individual sensing behaviors. The parameters are set as $P_{0}=40 \mathrm{~dB}$, $d_{0}=100 \mathrm{~km}, \lambda_{t h}=-15 d B$, and $m=6$ according to the previous results. The field $A$ is set to be a square with the side length being equivalent of $5 d B$ of the PU's signal attenuation. We assume that the real boundary is around the middle of the field, as the position of the real boundary has insignificant impacts on the sensing accuracy. For each setting, we conduct 100 independent runs and calculate the average.

5.2.1 Sensor Number \& Sensor Deployment. Here, we evaluate the sensing effectiveness of single channels $U_{k}$ under different deployments. Four sensor deployments are investigated: regular hexagon deployment, regular triangular, regular grid, and uniform random. Note that the first three are the only regular tessellations in 2-D spaces. We believe these four schemes can be representatives for other deployment schemes. For each deployment, we vary the sensor number $n_{k}$ from 25 to 900, and the resulting $U_{k}$ are shown in Figure 17. When calculating $U_{k}$, we disable the verification of the $P_{M D}$ and $P_{F A}$ to investigate the behavior of these schemes.

The results show that, in general, $U_{k}$ grows when more sensors are deployed for sensing. When the sensors are fewer than 200, the hexagon deployment outperforms the others, and the improvement is up to $56 \%$ compared with random deployment. When more sensors are used $\left(n_{k}>200\right)$, the triangle deployment gets more gain from more sensors and becomes the best. When more sensors are used, the improvement of the triangle deployment over others decreases to $9.8 \%$.

The sensing effectiveness of single channel $U_{k}$ is highly related to sensing accuracy, the $P_{M D}$ and $P_{F A}$. Figure 18 presents these two errors under different deployments. From this figure, we find that misdetection dominates the other, and both errors decrease as the sensor number increases. Given a mandatory requirement on the sensing accuracy, say $P_{M D}<0.1$ and $P_{F A}<0.1$, the triangular deployment needs $n_{k}>160$, the hexagon and uniform deployments need $n_{k}>200$ sensors, and the random deployment requires at least 300 sensors. These results indicate that the triangle deployment may be an attractive deployment scheme.

5.2.2 Rational of the Triangular Deployment. An interesting observation from the preceding experiments is that the averaged number of Voronoi neighbors does not have a direct connection with sensing accuracy (and thus $U_{k}$ ). This is counterintuitive as the Voronoi neighbors have a 


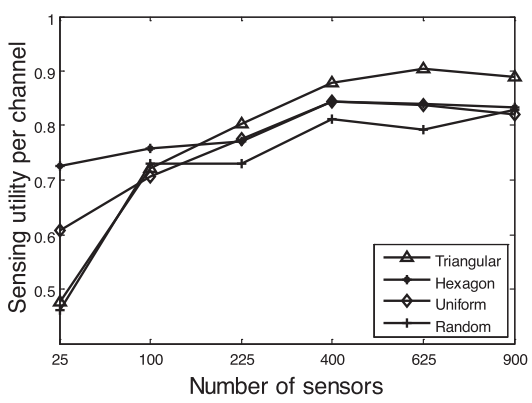

Fig. 17. Sensing effectiveness for single channels under different deployments.

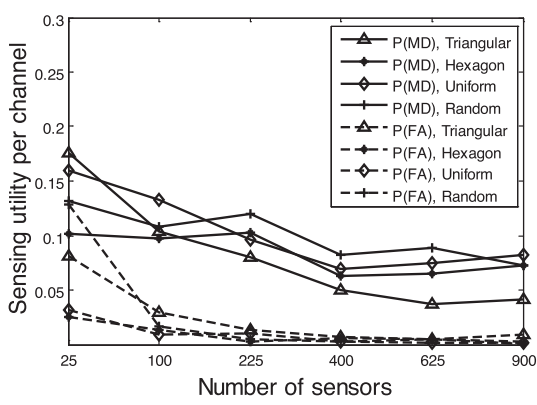

Fig. 18. Misdetection and false alarm percentage under different deployments.

critical role in our single channel spectrum sensing algorithm [39]. In the algorithm, a sensor will change its sensing results (say, "on") if more than half of its Voronoi neighbors have the opposite results (say, "off") by reciprocal calibration. Though intuitively more neighbors should get better calibrations, the grid deployment with four Voronoi neighbors does not always outperform the hexagon deployment with three neighbors. Indeed, it is always worse than the hexagon.

We make further investigations on this interesting observation. We find that, in our grid deployment, a sensor typically has five to six Voronoi neighbors due to the small disturbance we introduce to the positions of sensors. By this disturbance, sensors in the grid deployment will be likely to have a biased number of Voronoi neighbors in different directions. We argue that such biased Voronoi neighbors is the root cause of the performance degradation in the grid deployment. To justify this argument, we conduct more simulations. For the hexagon and triangular deployments, we modify the declaration algorithm, letting sensors calibrate their declarations only based on one side of their Voronoi-neighbors (e.g., only the left ones). The results depicted in Figure 19 confirm our argument. When sensors calibrate their declarations from half of their neighbors, sensing accuracy is demoted significantly. The sensing effectiveness of single channels decreases by nearly $25 \%$ for all scenarios. This argument to some extent explains why the random deployment cannot perform well in general.

5.2.3 Impact of the Number of Voronoi Neighbors. Another important observation is that the number of Voronoi neighbors also has great impact on $U_{k}$. In general, more neighbors will produce better sensing effectiveness as they will be more likely to give a correct calibration for each erroneous input. To justify this, we numerically study the impact of the number of Voronoi neighbors on the sensing results of individual sensors.

Suppose the sensed PU's power is $\overline{\mathrm{P}}$ at a sensor and the real power level is $P$. The threshold of the PU's covered area is $\lambda_{t h}$. Then, the probability that a sensor reports an "on" status before the calibration is $\operatorname{Pr}_{o n}=\operatorname{Pr}\left(\overline{\mathrm{P}} \geq \lambda_{t h}\right)$. The probability of an "off" report is $\operatorname{Pr}_{\text {off }}=\operatorname{Pr}\left(\overline{\mathrm{P}}<\lambda_{t h}\right)$. Suppose the node has $k$ Voronoi neighbors. After the calibration with these $k$ neighbors, the probability that the node reports a correct "on" status becomes:

$$
\operatorname{Pr}_{\text {on }}(k)=\sum_{i=\left\lceil\frac{k}{2}\right\rceil}^{k} C_{k}^{i} \operatorname{Pr}_{o n}^{i} \operatorname{Pr}_{\text {off, }}^{k-i}
$$

where $C_{k}^{i}$ is the choose function of $k$ and $i$. Similarly, the probability of a correct "off" status is:

$$
\operatorname{Pr}_{\text {off }}(k)=\sum_{i=\left\lceil\frac{k}{2}\right\rceil}^{k} C_{k}^{i} \operatorname{Pr}_{\text {off }}^{i} \operatorname{Pr}_{o n}^{k-i}
$$




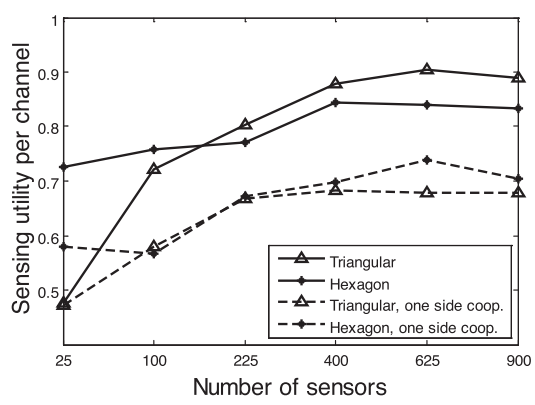

Fig. 19. Cooperation with all the Voronoi neighbors vs. with one side of the Voronoi neighbors.

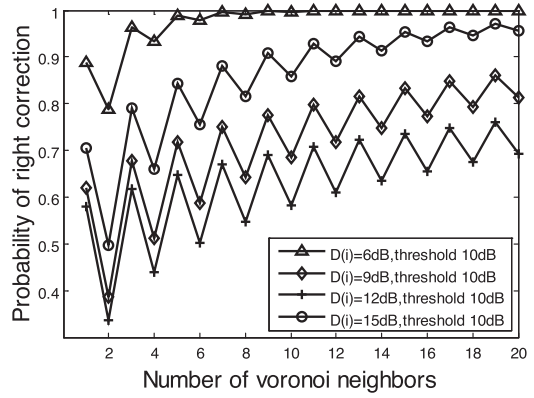

Fig. 20. Impact of the number of Voronoi neighbors.

Figure 20 depicts these two function. The plots have fluctuations because odd and even numbers of $k$ will perform very differently. From these numerical results, we find that a deployment of more Voronoi neighbors will favor calibration, and thus a higher sensing effectiveness of single channels is expected. Moreover, correct $P r_{o n}$ and $P r_{o f f}$ are not symmetrically equivalent and $P r_{o n}<$ $P r_{\text {off }}$ when other parameters are the same. That is the main reason why the misdetection errors dominate the false alarm errors, as we observed in Figure 18.

\subsection{Prototype Measurements}

In simulations, we assume that the Rayleigh model can adequately describe the sensing behavior of individual sensors. To justify this, we conduct a number of field studies and compare the real measurements with the theoretical models. Since there are no off-the-shelf spectrum sensors available, we use the $2.4 \mathrm{G}$ ISM band as the licensed band. We use a $802.11 \mathrm{~g}$ wireless router to emulate the behavior of PUs. Backlog traffic is injected so that the spectrum is very likely to be fully utilized. We use 10 Berkeley TelosB sensor nodes $[4,26]$ to function as spectrum sensors. They are configured in the same spectrum as that in the router and report the RSS to a laptop. Each sensor measures 1,000 samples of the spectrum, and the CDF of RSS at three locations are depicted. The signal strengths of these three locations are different; roughly $-10 \mathrm{db},-15 \mathrm{db}$, and $-20 \mathrm{db}$, respectively, and the sensing results are shown in Figure 16. Since we have no knowledge of the transmission power of the transmitter, we select a scaler such that the plots are comparable. Results show that the Rayleigh model can quite accurately characterize the sensing behavior. Though there are gaps between the field measurements and the models, there is a consistent trend.

\section{RELATED WORK}

Spectrum sensing at low signal power levels is crucially important in CRNs. The current research efforts can be mainly classified into two categories. One focuses on how to increase the individual sensing accuracy and reduce the probability of erroneous declarations. By path-loss propagation model, the RSS is a random variable with the mean related to the distance between the primary transmitter and the receiver [31]. Harmonic downmixing effect [22] was also studied, which may confuse the energy detector and should be taken into consideration. In addition to shadowing/multipath diversity, interference diversity was also explored [25]. For detection, various methods have been proposed. In additional to the simplest energy detector, feature detection [5] allowed users to distinguish the primary transmitter from SUs. Parameter sensitivity is studied in cognitive radio co-existance $[14,18]$. 
The other category of works looks for better cooperation schemes to produce better data fusion results. Distributed algorithms are proposed to estimate the boundary of no-talk PU regions [33, 34, 43]. Wang et al. [32] use the estimated locations of each SU rather than accurate locations. Clustering algorithms are also proposed to improve sensing efficiency and accuracy [6, 40]. Zhou et al. studied the participatory sensing paradigm for the event of arrival [45]. iSelf [30] attempted to label emotions with smartphones in cold-start using transfer learning algorithms, and Zheng et al. [44] detected ubiquitous smoking behaviors by employing radio CSI information. Zhou et al. [45] propose an arrival time prediction system based on participatory sensing. Commodity mobile phones are used as the basic sensing unit. A spatial-temporal opportunity detection method is proposed for spectrum-heterogeneous CRNs with two-dimensional sensing techniques [37]. Kim et al. [16] studied the scheduling of feature and energy detection, as well as forming sensor cluster to yield the best performance. Mobility issues are considered in mobile cognitive radios by opportunistic spectrum access [21]. Ji et al. [15] improves wireless channel utilization efficiency through a collision tolerance approach. Machine learning techniques are adopted in learning temporalspatial spectrum reuse [20, 42]. Ding et al. gave an overview of kernal-based learning for CRNs [9]. All these works are based on the traditional sensing devices of small-scale SUs. They do not consider the application scenarios of large fields. In such applications, the problem becomes to identify the boundary between the PU covered area and the other part.

Boundary detection has also been extensively studied. In existing boundary detection, the key issue is to derive a fit boundary based on correct inputs. A widely used tool is computational geometry. Some research efforts were devoted to recognizing boundaries between a sensor's covered and uncovered areas based on node degree [11], connectivity information [13], or solely on topology information [36]. In classifier-based approaches, it was assumed that sensor readings in different subareas were different. Accordingly, the data similarity could be a reliable indicator for the boundary. In particular, fault-tolerant detection was studied [17] and a distributed Bayesian algorithm was proposed. It allowed sensors to have error readings but focused on the off-boundary areas, where the erroneous sensors are always surrounded by correct ones. Multiple events were studied in follow-up works [1]. To conclude, none of the exiting work is suitable for our application scenarios where the erroneous inputs are notable and mixed together.

\section{CONCLUSION AND FUTURE WORK}

In this article, we proposed a new architecture of spectrum sensing in DSA. We adopt a dedicated SSN to provide a spectrum sensing service for end users. We described the general framework of this new SSN-enabled CRN and discussed the main research issues under this new service model. We focused on one of the problems and formulated it as boundary detection with notable sensing errors and proposed a cooperative boundary detection scheme to solve the problem.

We proved that cooperative boundary detection is asymptotically optimal. Experimental results showed that cooperative boundary detection could accurately derive the boundary in practice. Compared with traditional boundary detection, cooperative reduced detection errors by up to $95 \%$, with an average reduction of $85 \%$. Results also showed that cooperative boundary detection was more robust to erroneous input, making it particularly suitable for high-efficiency utilization of spectrum opportunities.

SSN-enabled CRNs are promising compared with the traditional sensing model in DSA, though many new and challenging issues arise. In this article, we only focus on one of the problemsSEM-and leave the others for future work. For example, we note a gap between real sensing and the theoretical models, as shown in Figure 16. For example, at the location of $-10 \mathrm{~dB}$, when signals are only $-20 \mathrm{~dB}$, the sensing probility should be as low as 0.1 . In practice, in more than $60 \%$, we 
detect the signals. And in all settings, the real sensing results are better than the model. This may need further investigations on more accurate and practical sensing models.

\section{REFERENCES}

[1] Torsha Banerjee, Bin Xie, and Dharma P. Agrawal. 2008. Fault tolerant multiple event detection in a wireless sensor network. Journal of Parallel and Distributed Computing 68, 9 (2008), 1222-1234. DOI : https://doi.org/10.1016/j.jpdc. 2008.04.009

[2] Zhipeng Cai, Shouling Ji, Jing He, and Anu G. Bourgeois. 2012. Optimal distributed data collection for asynchronous cognitive radio networks. In Proceedings of the of IEEE International Conference on Distributed Computing Systems. IEEE, 245-254.

[3] Lan Cheng, Chuanping Hu, Yunhuai Liu, and Lionel Ni. 2014. SCAS: Sensing channel assignment for wireless spectrum sensor networks. Wireless Networks 20, 5 (2014), 817-831.

[4] Chipcon. 2007. CC2420 Data Sheet. http://focus.ti.com/lit/ds/symlink/cc2420.pdf.

[5] Carlos Cordeiro, Kiran Challapali, and Monisha Ghosh. 2006. Cognitive PHY and MAC layers for dynamic spectrum access and sharing of TV bands. In Proceedings of the ACM TAPAS. ACM, New York, 3.

[6] Jingyi Dai and Shaowei Wang. 2017. Clustering-based spectrum sharing strategy for cognitive radio networks. IEEE fournal on Selected Areas in Communications 35, 1 (2017), 228-237.

[7] Mark de Berg, T. U. Eindhoven, K. Otfried Cheong, Marc van Kreveld, and Mark Overmars. 2008. Computational Geometry: Algorithms and Applications. Springer-Verlag.

[8] Guoru Ding, Jinlong Wang, Qihui Wu, Yu-Dong Yao, Fei Song, and Theodoros A. Tsiftsis. 2016. Cellular-base-stationassisted device-to-device communications in TV white space. IEEE fournal on Selected Areas in Communications 34, 1 (2016), 107-121.

[9] Guoru Ding, Qihui Wu, Yu-Dong Yao, Jinlong Wang, and Yingying Chen. 2013. Kernel-based learning for statistical signal processing in cognitive radio networks: Theoretical foundations, example applications, and future directions. IEEE Signal Processing Magazine 30, 4 (2013), 126-136.

[10] Xiaokun Fan and Jing Liu. 2010. MAC protocol for distributed relay-assisted cognitive radio networks. In Proceedings of the 6th International Conference on Wireless Communications Networking and Mobile Computing (WiCOM'10). IEEE, $11-14$.

[11] S. P. Fekete, A. Kroller, D. Pfisterer, S. Fischer, and C. Buschmann. 2004. Neighborhood-based topology recognition in sensor networks. In Proceedings of the International Symposium on Algorithms and Experiments for Sensor Systems, Wireless Networks and Distributed Robotics. Springer, 123-136.

[12] A. Ghasemi and E. S. Sousa. 2005. Collaborative spectrum sensing for opportunistic access in fading environments. In Proceedings of the IEEE International Symposium in Dynamic Spectrum Access Networks (DySPAN'05). IEEE, 131-136.

[13] R. Ghrist and A. Muhammad. 2005. Boundary recognition in sensor networks by topological methods. In Proceedings of the ACM/IEEE IPSN. 254-260.

[14] Andrea Hess, Francesco Malandrino, Nicholas J. Kaminski, Tri Kurniawan Wijaya, and Luiz A. DaSilva. 2016. Cognitive radio algorithms coexisting in a network: Performance and parameter sensitivity. IEEE Transactions on Cognitive Communications and Networking 2, 4 (2016), 381-396.

[15] Xiaoyu Ji, Yuan He, Jiliang Wang, Kaishun Wu, Daibo Liu, Ke Yi, and Yunhao Liu. 2017. On improving wireless channel utilization: A collision tolerance-based approach. IEEE Transactions on Mobile Computing 16, 3 (2017), 787-800.

[16] Hyoil Kim and Kang G. Shin. 2008. In-band spectrum sensing in cognitive radio networks: Energy detection or feature detection. In Proceedings of the ACM International Conference on Mobile Computing and Networking (Mobicom'08). ACM, New York,14-25. DOI : https://doi.org/10.1145/1409944.1409948

[17] Bhaskar Krishnamachari and Sitharama Iyengar. 2004. Distributed bayesian algorithms for fault-tolerant event region detection in wireless sensor networks. IEEE Transations on Computing 53, 3 (2004), 241-250. DOI : https://doi.org/10. 1109/TC.2004.1261832

[18] Mihir Laghate and Danijela Cabric. 2017. Cooperatively learning footprints of multiple incumbent transmitters by using cognitive radio networks. IEEE Transactions on Cognitive Communications and Networking 3, 3 (2017), $282-297$.

[19] Mo Li and Yunhao Liu. 2010. Rendered path: Range-free localization in anisotropic sensor networks with holes. IEEE/ACM Transactions on Networking (ToN) 18, 1 (2010), 320-332.

[20] J. Manco-Vásquez, S. Van Vaerenbergh, J. Vía, and Ignacio Santamaría. 2017. Kernel canonical correlation analysis for robust cooperative spectrum sensing in cognitive radio networks. Transactions on Emerging Telecommunications Technologies 28, 1 (2017), e2896.

[21] Alexander W. Min, Kyu-Han Kim, Jatinder Pal Singh, and Kang G. Shin. 2011. Opportunistic spectrum access for mobile cognitive radios. In Proceedings of the IEEE International Conference on Computer Communications (INFOCOM'11). IEEE, 2993-3001. 
[22] N. A. Moseley, E. Klumperink, and B. Nauta. 2008. A spectrum sensing technique for cognitive radios in the presence of harmonic images. In Proceedings of the IEEE International Symposium in Dynamic Spectrum Access Networks (DySPAN'08). 1-10. DOI: https://doi.org/10.1109/DYSPAN.2008.14

[23] Jorge Nocedal and Stephen J. Wright. 2006. Numerical Optimization. Springer-Verlag.

[24] IEEE 802.22 Working Group on Wireless Regional Area Networks. [n. d.]. http://www.ieee802.org/22/.

[25] Arash Parsa, Amin Aminzadeh Gohari, and Anant Sahai. 2008. Exploiting interference diversity for event-based spectrum sensing. In Proceedings of the IEEE International Symposium in Dynamic Spectrum Access Networks (DySPAN'08).

[26] Joseph Polastre, Robert Szewczyk, and David Culler. 2005. Telos: Enabling ultra-low power wireless research. In Proceedings of the ACM/IEEE IPSN.

[27] Stephen J. Shellhammer. 2008. Spectrum sensing in IEEE 802.22. In Proceedings of the International Workshop on Cognitive Information Processing (CIP'08). 9-15.

[28] Stephen J. Shellhammer, Sai Shankar N., Rahul Tandra, and James Tomcik. 2006. Performance of power detector sensors of DTV signals in IEEE 802.22 WRANs. In Proceedings of the International Workshop on Technology and Policy for Accessing Spectrum. ACM, 4-11.

[29] Carl R. Stevenson, Carlos Cordeiro, Eli Sofer, and Gerald Chouinard. 2005. Functional Requirements for the 802.22 WRAN Standard. http://www.ieee802.org/22/Meeting_documents/2005_Sept/22-05-0007-46-0000_RAN_ Requirements.doc.

[30] Boyuan Sun, Qiang Ma, Shanfeng Zhang, Kebin Liu, and Yunhao Liu. 2017. iSelf: Towards cold-start emotion labeling using transfer learning with smartphones. ACM Transactions on Sensor Networks (TOSN) 13, 4 (2017), 30.

[31] E. Visotsky, S. Kuffner, and R. Peterson. 2005. On collaborative detection of TV transmissions in support of dynamic spectrum sharing. In Proceedings of the IEEE International Symposium in Dynamic Spectrum Access Networks (DySPAN).

[32] Huaxia Wang and Yu-Dong Yao. 2018. Primary user boundary detection in cognitive radio networks: Estimated secondary user locations and impact of malicious secondary users. IEEE Transactions on Vehicular Technology 67, 5 (2018), 4577-4588.

[33] Tianyu Wang, Lingyang Song, Zhu Han, and Walid Saad. 2014. Distributed cooperative sensing in cognitive radio networks: An overlapping coalition formation approach. IEEE Transactions on Communications 62, 9 (2014), 31443160.

[34] Tsang-Yi Wang, Ming-Hsun Yang, and Jwo-Yuh Wu. 2016. Distributed detection of dynamic event regions in sensor networks with a Gibbs field distribution and gaussian corrupted measurements. IEEE Transactions on Communications 64, 9 (2016), 3932-3945.

[35] Xijun Wang, Tony Q. S. Quek, Min Sheng, and Jiandong Li. 2017. Throughput and fairness analysis of Wi-Fi and LTE-U in unlicensed band. IEEE fournal on Selected Areas in Communications 35, 1 (2017), 63-78.

[36] Yue Wang, Jie Gao, and Joseph S. B. Mitchell. 2006. Boundary recognition in sensor networks by topological methods. In Proceedings of the ACM Mobicom. 122-133.

[37] Qihui Wu, Guoru Ding, Jinlong Wang, and Yu-Dong Yao. 2013. Spatial-temporal opportunity detection for spectrumheterogeneous cognitive radio networks: Two-dimensional sensing. IEEE Transactions on Wireless Communications 12, 2 (2013), 516-526.

[38] Lei Yang, Yao Li, Qiongzheng Lin, Huanyu Jia, Xiang-Yang Li, and Yunhao Liu. 2017. Tagbeat: Sensing mechanical vibration period with COTS RFID systems. IEEE/ACM Transactions on Networking 25, 6 (2017), 3823-3835.

[39] Yanyan Yang, Yunhuai Liu, Qian Zhang, and Lionel Ni. 2010. Cooperative boundary detection for spectrum sensing using dedicated wireless sensor networks. In Proceedings of the IEEE INFOCOM. IEEE, 1-9.

[40] Alireza Zaeemzadeh, Mohsen Joneidi, Nazanin Rahnavard, and Guo-Jun Qi. 2018. Co-SpOT: Cooperative spectrum opportunity detection using bayesian clustering in spectrum-heterogeneous cognitive radio networks. IEEE Transactions on Cognitive Communications and Networking 4, 2 (2018), 206-219.

[41] Tengyi Zhang and Danny H. K. Tsang. 2011. Optimal cooperative sensing scheduling for energy-efficient cognitive radio networks. In Proceedings of the IEEE INFOCOM. IEEE, 2723-2731.

[42] Yi Zhang, Wee Peng Tay, Kwok Hung Li, Moez Esseghir, and Dominique Gaïti. 2016. Learning temporal-spatial spectrum reuse. IEEE Transactions on Communications 64, 7 (2016), 3092-3103.

[43] Yi Zhang, Wee Peng Tay, Kwok Hung Li, and Dominique Gaïti. 2014. Distributed boundary estimation for spectrum sensing in cognitive radio networks. IEEE fournal on Selected Areas in Communications 32, 11 (2014), 1961-1973.

[44] Xiaolong Zheng, Jiliang Wang, Longfei Shangguan, Zimu Zhou, and Yunhao Liu. 2017. Design and implementation of a CSI-based ubiquitous smoking detection system. IEEE/ACM Transactions on Networking (2017).

[45] Pengfei Zhou, Yuanqing Zheng, and Mo Li. 2012. How long to wait?: Predicting bus arrival time with mobile phone based participatory sensing. In Proceedings of the 10th International Conference on Mobile Systems, Applications, and Services. ACM, 379-392.

Received April 2018; revised August 2018; accepted September 2018 